\title{
Supporting the Continuum of Care for Combat Wounded Patients: Adaptive Interfaces for Personal Health Records
}

\author{
Harry D. Tunnell IV and Aeshvarya Verma \\ Grappa Lab, Indiana University School of Informatics (Indianapolis) \\ hadtunneaiupui.edu
}

\begin{abstract}
In this paper, we describe a concept for an adaptive interface for a military Personal Health Record (PHR). PHRs are electronic records used by people to manage their personal healthcare information. In the Military Healthcare System, combat wounded patients encounter a range of challenges due to the unique nature of the military environment and the severity of their wartime injuries. These factors affect how people interact with a computer interface. In many instances, combat wounded patients eventually have assistance from family members, professional caregivers, and others. This forms a disparate enduser population. Because the pool of potential users includes people with a wide range of cognitive and physical capabilities, an adaptive interface that considers attributes of health can improve user experiences.
\end{abstract}

Keywords: Adaptive interfaces, ability-based design, Personal Health Record (PHR), combat wounded, military informatics.

\section{Introduction}

In order to meet the needs of disparate users within the Military Healthcare System, the concept to provide multiple unique interface options to access one person's Personal Health Record (PHR) is relevant. Devising PHRs to support different types of user has been identified as an important and critical challenge of system design [1]. To overcome the challenge of accessibility to a PHR, we propose that a single record have multiple interfaces based upon user cognitive and physical ability. The users envisioned for this PHR are people who support the continuum of care for wounded in action (WIA) military personnel such as patients, family members, and professional caregivers.

Traditional human-computer interaction (HCI) approaches include examining individual differences, but not in the context of single patient multi-user systems with multiple interfaces. Understanding individual differences is important for HCI models to improve a single interface and experiences for multiple users, design adaptive interfaces that change based upon the interaction of a single user, or to recognize patterns of search interaction to improve web search [2,3]. Our proposed approach is different because it suggests multiple interfaces for a single system based upon 
individual differences. The objective is to deliver the right interface to users based upon the specific end-user's ability to interact with the system. For example, patients may use an interface based upon ability-based designs while family members and caregivers rely on more typical user-centered designs. Here we outline the role of PHRs in this context, highlight the military informatics environment that defines usability requirements, and describe a concept for a PHR adaptive interface.

\section{Personal Health Records to Support Combat Wounded}

\subsection{The Role of Personal Health Records}

There are various types of electronic records within the healthcare domain. PHRs and Electronic Health Records (EHRs) are among the most common. Each is used to manage patient information, but they have different roles and audiences. The PHR is typically designed for one person who can grant access to others. PHRs are webbased applications that people use to manage and share their health data or access the records of others (as long as they have the proper credentials) [4, 5]. PHRs are commercial (e.g., Medefile, Microsoft HealthVault) or institutional (e.g., MyHealtheVet) products. The EHR, on the other hand, is a repository of computer processable information that can be securely transmitted and that multiple users can use to access information about a single patient [6]. An EHR is used by medical professionals and is accessed using an institutional system (hospital, medical practice, clinic, etc.) [4].

\subsection{The Military Informatics Environment}

There have been nearly 50,000 combat wounded service members since September 11, 2001 [7]. Military professionals realized that traditional information systems to track patients were inadequate due to the pace of combat operations, casualty acuity, and casualty load in a complex healthcare environment [8]. Military informatics is the design of information systems (IS) for a military audience to accomplish militaryrelated goals. A military informatics based PHR, incorporating interfaces that change based upon end-user cognitive and/or physical capability, can help people navigate the complex Military Healthcare System.

Wounded personnel within the Department of Defense (DOD) receive treatment at the point of injury and are evacuated to an acute care facility in the combat theater $[8$, 9]. The next evacuation center is often a non-theater hospital such as Landsthul, Germany [8-10]. Patients are then evacuated to medical facilities in the U.S. or returned to their military unit. Patients are transferred to a different medical system such as the Veteran's Administration (VA) upon discharge or medical retirement [10]. Solutions such as the Joint Patient Tracking Application, which tracks real-time patient status, have been implemented as a result of the volume of wounded patients and complexity of the military healthcare environment [8].

The military; however, has yet to implement a comprehensive patient-facing IS, preferring to incorporate PHR-like features into existing systems. For example, the U.S. Army includes deployment health information in its Army Knowledge Online 
(AKO) application, but soldiers also use the system for e-mail, training and clothing records, and other aspects of military information management. AKO is a document based system that requires users to have domain knowledge about U.S. Army organization and culture which makes navigation and searches difficult for newcomers and outsiders [11]. Relying on AKO in lieu of a PHR fails to recognize that AKO is not intuitive and requires patients to recall complicated domain specific information during periods of cognitive impairment due to injury or medication. Family members and caregivers likely do not have the requisite domain knowledge to find patient health information within AKO. A PHR with an adaptive interface can provide important tools to support the continuum of care for combat wounded patients.

Combat wounds often result from an adversary's employment of advanced weapons and munitions typically not available to civilians (e.g., an Explosively Formed Penetrator, which is commonly known as an EFP). Despite technical advances in weaponry, American military personnel are less likely to be killed in action today than earlier wars due to improved protective equipment, early damage-control surgery, and rapid evacuation out of the combat theater [9]. There has been a reduction in patient mortality from $22.8 \%$ during WWII to $8.8 \%$ today [8]. Even formerly catastrophic injuries are survivable; four military patients endure as quadruple amputees [12]. The fact that people live with increased cognitive and physical impairment means they may rely upon a network of family and professional caregivers for help. This is complicated by the international and cross-healthcare system nature of military health information management. The need for a military informatics derived multiuser PHR within the defense medical ecosystem is essential.

\subsection{Information Sharing and Accessibility}

Even though PHRs are designed primarily for a patient to manage their own data, many have features that allow healthcare providers to add data to the record. Furthermore, some commercial PHRs have built-in functions that allow a person to share their PHR outside of the medical community [13]. Usability testing consistently shows people want the ability to share PHR information with medical providers and others outside of the healthcare profession $[1,5,14]$. This also supports the concept of a PHR for one patient that can be shared among several people.

About 70 million people in the U.S. use some form of PHR [4]. Despite the popularity of PHRs, testing indicates that significant usability problems remain [1]. Recent innovations regarding PHRs tend to focus on security, end-user privacy, and content. Fortunately, the scope of PHR innovation is expanding to include aspects such as intelligence. The goal of an intelligent PHR is to automatically provide users with personalized healthcare information [15]. Additionally, research by Liu, Shih [1] indicates that PHRs should consider accessibility designs so that end-users with disabilities can customize the system to suit their specific needs. In the next section, we examine customization as a means to support combat wounded patient PHR needs. 


\section{Discussion}

\subsection{End-User Types}

One of the challenges of design is having the right information about users [16]. Demographic information for combat wounded personnel such as age, race, gender, and injury is highly documented and simplifies data collection because there is precision in the knowledge about the wounded population [17]. For example, there were 1,286 amputations during the period 2001-2009 [17]. An amputee registry has been created to track and manage military patient care and needs, which tend to be different from the needs of elderly patients who lose a limb due to vascular disease [10]. Traumatic Brain Injury (TBI) has a greater rate of injury than amputation with 43,779 personnel diagnosed between 2003 and 2007 [17]. Unfortunately, research to support designs for cognitively dysfunctional users is inadequate [16]. Yet the rate of TBI compared to other wounds highlights an immediate need to support cognitively impaired users. In addition to the wounded population of end-users, family members and caregivers who are not disabled are potential users. Traditional HCI methods of interface design are acceptable for this population.

\subsection{Interface Customization}

Interface customization has become a normal part of the user experience. It is common in both client-side and server-side applications. Marathe and Sundar [18] define interface customization as an activity that a user performs to change the presentation and functionality of an interface to increase its personal relevance. Customization is increasingly popular because it is tied to the user's sense of identity and sense of control-as many as $92 \%$ of research participants customize an application in some manner $[18,19]$. Even though there is high acceptance of customization, user choices are typically limited because customization is a difficult programming task and requires significant extra work for developers [19]. There are generally two options for customization: functional and cosmetic. Marathe [20] notes that functional customization pertains to work practices and cosmetic customization pertains to embellishments surrounding the work practices. Customization can improve user experiences for family members and caregivers. Unfortunately, customization does not support the wide disparity in cognitive and physical ability common among the combat wounded population. Fortunately, adaptive interface capabilities can go beyond customization.

\subsection{Adaptive Interfaces}

Adaptive user interfaces present a number of solutions that can be matched to the changeability of users and the environment [21]. This is significantly different from an interface that presents a single solution for multiple users. For example, a Microsoft Windows interface that emphasizes WIMP-style (windows, icons, menu, and pointer) interaction through a graphical user interface (GUI) and allows users to modify the color, size, and position of backgrounds and icons is customizable but not 
adaptive. The Microsoft Windows 8 GUI, on the other hand, lets users transition between WIMP and tile touch screen-this an example of an interface that has characteristics of being customizable and adaptive.

Even though interfaces that employ WIMP, touch screen, speech, gesture, and other forms of interaction are not new, they are increasingly available to the public on commercial personal use systems. However, the end-user must manually select the style of interaction. Montague [22] proposed a framework that provides a user with the most suitable interaction experience based upon their needs at the time they operate the interface. In the framework, web services update the client model without requiring calibration exercises or manual configuration of the interface [22]. This approach could have utility for PHR adaptive interfaces.

The typical approach to adaptive interfaces and users with disabilities has been to focus almost exclusively on accessibility concerns or interfaces that run on different devices (e.g., mobile or desktop) [23]. Regarding specific disability, Newell [16] states that blindness is the priority in accessibility research and this has resulted in a lack of support for the large population of people with other disabilities. The approach proposed herein is a multimodal presentation of interfaces based upon users that may or may not have a disability. This allows a user to employ an interaction option based upon their physical condition, mental state, or level of medication for instance. Additionally, an adaptive interface that incorporates intelligence can recommend modifications to the patient based upon the healthcare data managed within the PHR. For example, once data about a change in medication is entered into the PHR, the intelligence feature presents the patient with interface options based upon how the medication affects cognition (e.g., changing from Percocet to Ibuprofen).

Ability-based design is a new concept that offers opportunities to improve the user experience for wounded personnel. Rather than focus on user disability (the dominant approach), ability-based designs emphasize what a person can do and the system is designed around their capability to interact with an IS rather than an inability to use hardware and software [24]. Multimodal interfaces that consider end-user ability rather than disability allow a user to select, or have presented to them, the interaction style they prefer to use for their tasks [25]. A library of adaptive interfaces can include designs by modality for a specific disability. The library provides an integrated yet changeable solution for a single IS with multiple users based upon cognitive and physical ability rather than aesthetic preferences or workflow.

An interface library is important because the patient's capability may change as they undergo treatment and rehabilitation. For example, a person who survives an EFP attack may suffer multiple injuries resulting in amputation and a diagnosis of TBI. Their cognition might be affected initially by the explosion and/or powerful pain medications. Speech recognition technology is relevant in this case because speech interaction is important for people with cognitive disabilities who have difficulty remembering the mapping of menu items [25]. As the patient's medication and capability changes based upon cognitive improvement, lack of chronic pain, use of prosthesis, or successful limb transplantation surgery, they may wish to modify their interaction style. Customization does not support dramatic fluctuations in patient ability but a library of interfaces based upon impairment type, matched with interaction style, may 
improve the user experience. Furthermore, an ability-based methodology promotes long-term adaptive approaches (e.g., when a patient transitions from treatment to rehabilitation the interface is modified to reflect enhanced ability).

Yahoo!, an early leader in interface personalization, developed a massive User Database (USB) to support scalability and improve the speed and efficiency of personalization [26]. Designers of a PHR for combat wounded, on the other hand, potentially have access to details about the complete wounded population and thus have an opportunity to design interfaces accordingly. Once interfaces are implemented using a server-side distributed system, a dialogue record that contributes to the interaction knowledge base can be maintained for each user interaction [21]. In a manner reminiscent of the UDB, as the interaction knowledge base grows, changes are made to the library [21]. In this way, designers learn from actual user interaction with the system.

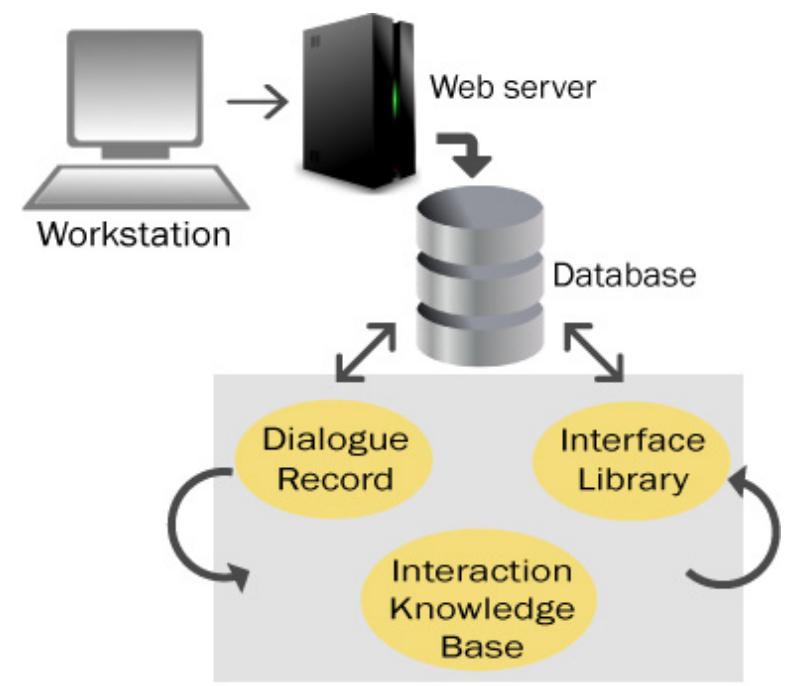

Fig. 1. A PHR adaptive interface architecture example

\subsection{Implications for Design}

Accessibility for web based technologies is considered an important part of the design process by HCI professionals [27]. Unfortunately, the actual implementation of accessibility features is limited, and visual impairment is the most common disability considered in accessibility designs [16, 27]. The lack of implementation and the focus on visual impairment does not meet the needs of the population of young combat wounded patents who will require extensive long-term care.

In addition to advances in technology and better knowledge about the military disabled population, it is important for designers to have better access to military patients during development. A major problem of interface design for assistive technologies is that it is difficult to recruit disabled people to test a new design [28]. The nature of 
military service allows commanders to make some decisions about testing. This means that leaders within DOD may be able to authorize and direct usability testing.

\section{$4 \quad$ Limitations and Future Work}

Despite the fact that there is a significant number of healthcare IS within DOD that store and retrieve documentation about casualties, there is not a comprehensive and coherent single user interface available to access the multitude of legacy systems, so there are limitations to the quality of casualty information [9]. Fortunately, the VA has been a leader in PHR development and MyHealtheVet (the VA's web-based PHR) has undergone usability testing with older veterans [5]. This provides a frame of reference to understand the needs of current veterans and wounded personnel.

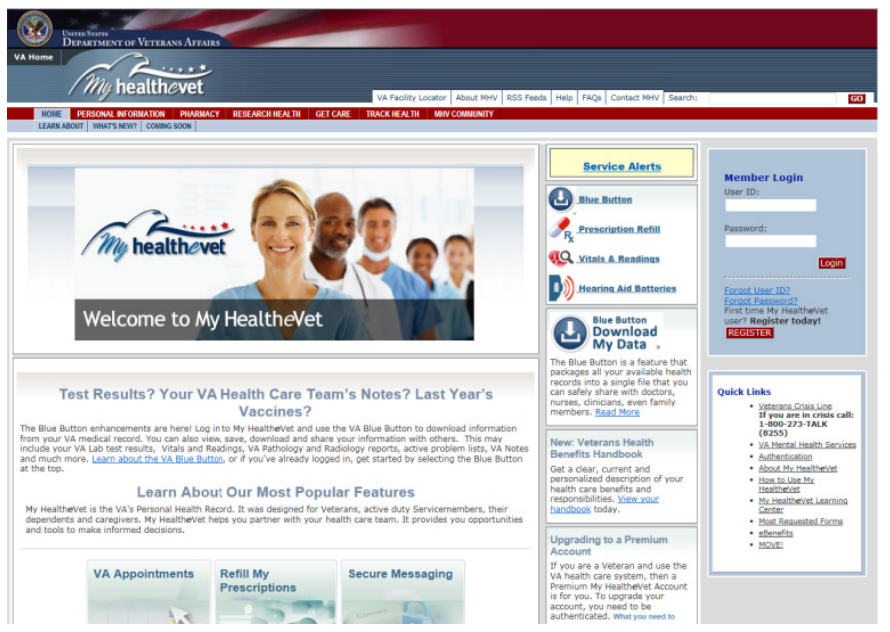

Fig. 2. The MyHealtheVet interface

\section{Conclusion}

Disabled users are often overlooked during design and testing [25]. A PHR for a military audience needs to include the combat wounded population early. A framework that incorporates military informatics and HCI can lead to adaptive designs that provide satisfactory user experiences for combat wounded personnel as they progress through the continuum of care.

It is only recently that non-cost prohibitive technology that is reliable, easy to use, and includes multimodal input devices on a single system has become widely available to the public. This offers new possibilities to design for a better user experience. Adaptive interfaces improve accessible technology by identifying user needs and providing a dynamically personalized user interface [23]. These ideas extend to the civilian community because people often have multiple disabilities - UK government 
research indicates that $80 \%$ of people with a sensory disability (e.g., visual) also have another disability (e.g., motor) [29].

Acknowledgements. The authors thank Jason Saleem for providing feedback on the initial concept and Mark Pfaff and Michael Lewis for commenting on drafts of the paper.

\section{References}

1. Liu, L.S., Shih, P.C., Hayes, G.R.: Barriers to the adoption and use of personal health record systems. In: Proceedings of the 2011 iConference, pp. 363-370. ACM, Seattle (2011)

2. Benyon, D.: Accommodating individual differences through an adaptive user interface, Computing Department. Open University, Milton

3. Buscher, G., et al.: Large-scale analysis of individual and task differences in search result page examination strategies. In: Proceedings of the Fifth ACM International Conference on Web Search and Data Mining, pp. 373-382. ACM, Seattle (2012)

4. Señor, I.C., Alemán, J.L.F., Toval, A.: Personal Health Records: New means to safely handle health data? Computer 45(11), 27-33 (2012)

5. Haggstrom, D.A., et al.: Lessons learned from usability testing of the VA's personal health record. Journal of the American Medical Informatics Association 18(suppl. 1), i13-i17 (2011)

6. Sachdeva, S., Bhalla, S.: Semantic interoperability in standardized electronic health record databases. Journal Data and Information Quality 3(1), 1-37 (2012)

7. Department of Defense, U.S. Casualty Stats. US Government, Washington, DC (2012)

8. Eastridge, B.J., et al.: Trauma system development in a theater of war: Experiences from Operation Iraqi Freedom and Operation Enduring Freedom. The Journal of TRAUMA Injury, Infection, and Critical Care 61(6), 1366-1373 (2006)

9. Gerhardt, R.T., et al.: Out-of-hospital combat casualty care in the current war in Iraq. Annals of Emergency Medicine 53(2), 169-174 (2009)

10. Peake, J.B.: Beyond the Purple Heart - Continuity of care for the wounded in Iraq. The New England Journal of Medicine 352(3), 219-222 (2005)

11. Douglas, L.: An object and performance framework for implementation of web-based knowledge sharing technology. Journal of Theoretical and Applied Electronic Commerce Research 4, 57-71 (2009)

12. Ruane, M.E.: Johns Hopkins Hospital performs double arm transplant on Army soldier. The Washington Post (2013)

13. Martino, L., Ahuja, S.: Privacy policies of personal health records: An evaluation of their effectiveness in protecting patient information. In: Proceedings of the 1st ACM International Health Informatics Symposium, pp. 191-200. ACM, Arlington (2010)

14. Baird, A., North, F., Raghu, T.S.: Personal Health Records (PHR) and the future of the physician-patient relationship. In: Proceedings of the 2011 iConference, pp. 281-288. ACM, Seattle (2011)

15. Luo, G., Tang, C., Thomas, S.B.: Intelligent personal health record: Experience and open issues. In: Proceedings of the 1st ACM International Health Informatics Symposium, pp. 326-335. ACM, Arlington (2011) 
16. Newell, A.F.: Accessible computing - past trends and future suggestions: Commentary on Computers and People with Disabilities. ACM Transaction in Accessible Computing 1(2), $1-7(2008)$

17. Fischer, H.: United States military casualty statistics: Operation Iraqi Freedom and Operation Enduring Freedom, C.R. Service, Editor, US Government: Washington, DC (2009)

18. Marathe, S., Sundar, S.S.: What drives customization?: Control or identity? In: Proceedings of the SIGCHI Conference on Human Factors in Computing Systems, pp. 781-790. ACM, Vancouver (2011)

19. Lee, L.-C., Lutteroth, C., Weber, G.: Improving end-user GUI customization with transclusion. In: Proceedings of the Thirty-Third Australasian Conference on Computer Science, vol. 102, pp. 163-172. Australian Computer Society, Inc., Brisbane (2010)

20. Marathe, S.S.: Investigating the psychology of task-based and presentation-based UI customization. In: Extended Abstracts on Human Factors in Computing Systems, CHI 2009, pp. 3129-3132. ACM, Boston (2009)

21. Benyon, D.: Adaptive systems: A solution to usability problems, Computing Department. Open University, Milton

22. Montague, K.: Interactions speak louder than words: Shared user models and adaptive interfaces. In: Adjunct Proceedings of the 25th Annual ACM Symposium on User Interface Software and Technology, pp. 39-42. ACM, Cambridge (2012)

23. Peissner, M., et al.: MyUI: Generating accessible user interfaces from multimodal design patterns. In: Proceedings of the 4th ACM SIGCHI Symposium on Engineering Interactive Computing Systems, pp. 81-90. ACM, Copenhagen (2012)

24. Wobbrock, J.O., et al.: Ability-Based Design: Concept, Principles and Examples. ACM Transactions on Accessible Computing 3(3), 9:1-9:27 (2011)

25. Kortum, P. (ed.): HCI beyond the GUI: Design for haptic, speech, olfactory, and other nontraditional interfaces. Morgan Kaufmann Publishers, Boston (2008)

26. Manber, U., Patel, A., Robison, J.: Experience with personalization of Yahoo! Communications of the ACM 43(8), 35-39 (2000)

27. Putnam, C., et al.: How do professionals who create computing technologies consider accessibility? In: Proceedings of the 14th International ACM SIGACCESS Conference on Computers and Accessibility, pp. 87-94. ACM, Boulder (2012)

28. Mankoff, J., Hayes, G.R., Kasnitz, D.: Disability studies as a source of critical inquiry for the field of assistive technology. In: Proceedings of the 12th International ACM SIGACCESS Conference on Computers and Accessibility, pp. 3-10. ACM, Orlando (2010)

29. Kienle, H., et al.: Ten years of access for all from WSE 2001 to WSE 2011. In: 13th IEEE International Symposium on Web Systems Evolution (2011) 